\title{
A CHARACTERIZATION OF DISTAL AND POINT-DISTAL MINIMAL TRANSFORMATION GROUPS ${ }^{1}$
}

\author{
JOSEPH F. KENT
}

\begin{abstract}
If $G$ is a locally compact topological group, let $B C(G)$ denote the set of real-valued, bounded, uniformly continuous functions on $G$ with the compact-open topology. Using the fact that the distal (weakly distal) functions are the elements of $B C(G)$ whose orbit closures are compact distal (point-distal) minimal sets, we can characterize compact distal and point-distal minimal transformation groups. Let $(X, G, \phi)$ be a right transformation group where $X$ is compact Hausdorff and minimal under $G$. Then $X$ is a compact distal (point-distal) minimal set if and only if there is a point $x \in X$ such that for any homomorphism $h$ : $X \rightarrow B C(G), h(x)$ is a right distal (weakly distal) function.
\end{abstract}

1. Introduction. The classification of minimal sets is one of the goals of topological dynamics. We will be concerned with three types of compact minimal sets: equicontinuous, distal, and point-distal. In [3] Baum established necessary and sufficient conditions for the abelian topological group $G$ to be equicontinuous on the compact Hausdorff minimal set $X$ where $(X, G, \phi)$ is a transformation group. Ellis [6] showed the result valid in the nonabelian case. Their work generalizes an unpublished theorem of Kakutani for the case where $G$ is the reals and $X$ is a compact metric space.

Theorem [KaKutani-Baum-Ellis]. Let $(X, G, \phi)$ be a right transformation group where $X$ is compact Hausdorff and minimal under $G$. Then $G$ is equicontinuous provided there exists $x \in X$ such that for all $F \in C(X), F_{x}=F \circ \phi_{x}$ is a right von Neumann almost periodic function.

This paper will establish similar results for compact distal and pointdistal minimal sets using dynamical characterizations of distal and weakly distal functions.

Received by the editors April 15, 1971.

AMS 1970 subject classifications. Primary 54H15, 54H20; Secondary 54C35.

Key words and phrases. Distal transformation group, point-distal transformation group, distal function, weakly distal function, von Neumann almost periodic function, functions coming from a transformation group.

${ }^{1}$ This research is part of a dissertation for the degree of Doctor of Philosophy at the University of Virginia.

(c American Mathematical Society 1972 
2. Definitions. For the standard definitions and notation of topological dynamics the reader is referred to [8]. We will be using uniform spaces in the following and $x \alpha$ will denote the set $\{y \in X:(x, y) \in \alpha\}$ where $x \in X$ and $\alpha$ is an index of $X$. For properties of uniform spaces see [4].

A right transformation group $(X, G, \phi)$ where $X$ is a uniform space is said to be right equicontinuous, or $G$ is right equicontinuous on $X$, if the collection $\left\{\phi_{g}: g \in G\right.$, where $\left.\phi_{g}(x)=\phi(x, g)\right\}$ is equicontinuous at each point of $X$. If $(X, G, \phi)$ is a transformation group where $X$ is a uniform space, then a point $x \in X$ is said to be a distal point provided that for any $y \in X$ with $x \neq y$, there is an index $\alpha$ of $X$ such that $y g \notin(x g) \alpha$ for all $g \in G$. The symbol $x g$ is the usual notation for $\phi(x, g) .(X, G, \phi)$ or $X$ is said to be distal if every point is a distal point. A transformation group is pointdistal if there is a distal point whose orbit closure is the whole space. Ellis [5] began to study distal transformation groups because they exhibited many characteristics similar to those where $G$ is equicontinuous. Every compact equicontinuous minimal set is distal, and clearly every distal minimal set is point-distal. Also, all compact point-distal transformation groups are minimal, but there are compact minimal sets which contain no distal points. Further properties of these minimal sets may be found in [7].

For topological properties of the function spaces to be introduced, one is referred to [4]. Let $R$ denote the real numbers. The set $B U(G)$ consists of the real-valued, bounded, uniformly continuous functions on $G$ with the topology of uniform convergence. One may form the right transformation group $(B U(G), G, \theta)$ by defining $\theta: B U(G) \times G \rightarrow B U(G)$ by $\theta(F, g)=F_{g}$ where $F_{g}(h)=F(g h)$ for $g, h \in G, F \in B U(G)$. Let $C(G)$ denote the real-valued continuous functions on $G$ with the compact-open topology. If $G$ is locally compact, one may form the right transformation group $(C(G), G, \psi)$ where $\psi$ is defined in a manner similar to $\theta$. The functions in $C(G)$ whose orbit closures are compact are precisely the bounded uniformly continuous functions. Let $B C(G)$ denote the real-valued, bounded, uniformly continuous functions on $G$ with the compact-open topology. By restricting $\psi$ to $B C(G) \times G$ one has another right transformation group. For results concerning the relationships among these transformation groups, one is referred to [1] and [2].

3. Functions coming from transformation groups. A function $f \in B C(G)$ is said to come from the compact transformation group $(X, G, \phi)$ if there is a homomorphism $h: X \rightarrow B C(G)$ of transformation groups with $f \in h(X)$. Every such homomorphism $h$ may be written as $h(x)=F \circ \phi_{x}$ for all $x \in X$ where $F$ is some real-valued continuous function on $X$. Conversely, any such function $F$ determines a homomorphism $x \rightarrow F \circ \phi_{x}$ of $(X, G, \phi)$ into $(B C(G), G, \psi)$. 
A function $f: G \rightarrow R$ is von Neumann right almost periodic provided its orbit closure is a compact subset of $B U(G)$. Auslander and Hahn [2] established the following theorem when $G=R$ which provides a dynamical characterization in $B C(G)$ of almost periodic functions. The proof is essentially the same for locally compact groups $G$ and is omitted.

THEOREM 3.1. Let $G$ be a locally compact topological group. Then the following statements are pairwise equivalent:

(i) $f: G \rightarrow R$ is a von Neumann right almost periodic function;

(ii) $f$ comes from a compact right equicontinuous minimal transformation group;

(iii) the closure of the orbit of $f$ in $B C(G)$ is a compact right equicontinuous minimal set.

As $B C(G)$ consists of the functions in $C(G)$ with compact orbit closures, any function $f \in C(G)$ which comes from a compact right transformation group is in $B C(G)$. Part (ii) of the theorem along with this fact motivated L. Auslander and Hahn [2] to introduce and study the distal functions. A continuous function $f: G \rightarrow R$ is said to be right distal if it comes from some compact distal right transformation group. The algebra of distal functions has been extensively studied by Knapp [9]. In [1] a right weakly distal function is defined as one which comes from a distal point of a compact point-distal right transformation group. It is clear that the class of von Neumann right almost periodic functions is contained in the class of right distal functions which is in turn contained in the right weakly distal functions. When $G=R$ these inclusions are all proper. With the help of a lemma of Veech [10] we characterize the right distal and weakly distal functions by means of the dynamical properties of their orbit closures in $B C(G)$. These results may be found in [1] and [2] for the case $G=R$.

LemMA 3.2 [VeEch]. Let $h:(X, G, \phi) \rightarrow(Y, G, \theta)$ be a homomorphism of compact right transformation groups. For each distal point $x \in X, h(x)$ is a distal point of $h(X) \subseteq Y$.

THEOREM 3.3. Let $G$ be a locally compact topological group and $f \in B C(G)$. Then $f$ is a right distal function if and only if the orbit closure of $f$ in $B C(G)$ is a compact distal minimal set.

Proof. Let $\operatorname{cl}[f G] \subseteq B C(G)$ denote the closure of the orbit of $f$. If $\operatorname{cl}[f G]$ is a compact distal minimal set, then the inclusion map $i: \operatorname{cl}[f G] \rightarrow$ $B C(G)$ is a homomorphism of right transformation groups and $f \in i(\operatorname{cl}[f G])$. Thus $f$ is a right distal function.

Conversely, suppose $f$ is a right distal function. Then $f$ comes from some compact distal minimal right transformation group $(X, G, \phi)$ 
or, equivalently, there is a homomorphism $h: X \rightarrow B C(G)$ of right transformation groups with $f \in h(X)$. Now $\operatorname{cl}[f G] \subseteq h(X)$, because $h(X)$ is closed and invariant. Since $h(X)$ is also minimal, being the homomorphic image of a compact minimal set, we see that $\operatorname{cl}[f G]=h(X)$. By the lemma, $h(X)=\operatorname{cl}[f G]$ consists of distal points, and $\operatorname{cl}[f G]$ is a compact distal minimal set.

THEOREM 3.4. Let $G$ be a locally compact topological group and $f \in B C(G)$. Then $f$ is a right weakly distal function if and only if $f$ is a distal point of $\mathrm{cl}[f G] \subseteq B C(G)$.

We omit the proof which is similar to the proof of Theorem 3.3.

4. Main results. The work of Baum and Ellis is done without the assumption that $G$ is locally compact since they are able to use a dynamical characterization in $B U(G)$ of almost periodic functions. We must rely on Theorems 3.3 and 3.4 when considering distal and weakly distal functions and hence must assume that $G$ is locally compact in order to establish corresponding theorems for compact distal and point-distal minimal right transformation groups.

THEOREM 4.1. Let $(X, G, \phi)$ be a right transformation group where $X$ is compact Hausdorff, $G$ is a locally compact topological group, and $X$ is minimal under $G$. Then $X$ is a compact distal minimal set if and only if there is a point $x \in X$ such that for any homomorphism $h: X \rightarrow B C(G), h(x)$ is a right distal function.

Proof. If $X$ is a compact distal minimal set, then for every homomorphism $h: X \rightarrow B C(G), h(X)$ consists, by definition, of right distal functions. Hence any point $x \in X$ will satisfy the theorem.

Conversely, suppose there is a point $x \in X$ such that $h(x)$ is a right distal function for all homomorphisms $h: X \rightarrow B C(G)$. Then $h(X)=\operatorname{cl}[h(x) G]$ is a compact distal minimal set for any such $h$ by Theorem 3.3. Let $y \in X$. We claim that $y$ is a distal point. Suppose $z \in X$ with $z \neq y$. There is a continuous function $F \in C(X)$ such that $F(y) \neq F(z)$. Let $h: X \rightarrow B C(G)$ be the homomorphism of transformation groups given by $h(x)=F \circ \phi_{x}$. Then $h(y) \not \equiv h(z)$ since $h(y)(e)=F(y) \neq F(z)=h(z)(e)$ where $e$ is the identity element of $G$. As $h(X)$ is a distal minimal set there is an index $\beta$ of $h(X)$ such that $h(z) g \notin(h(y) g) \beta$ for all $g \in G$. Let $\alpha=(h \times h)^{-1}(\beta)=$ $\left\{\left(x_{1}, x_{2}\right) \in X \times X:\left(h\left(x_{1}\right), h\left(x_{2}\right)\right) \in \beta\right\}$. Since $h$ is uniformly continuous, $\alpha$ is an index of $X$. Now $z g \notin y g \alpha$ for all $g \in G$. For if $z g \in y g \alpha$ for some $g \in G$, then $h(z g) \in h(y g) \beta$. As $h$ is a homomorphism, this implies $h(z) g \in(h(y) g) \beta$ which is a contradiction to the choice of $\beta$. Thus $y$ is a distal point, and $X$ is a compact distal minimal set. 
THEOREM 4.2. Let $(X, G, \phi)$ be a right transformation group where $X$ is compact Hausdorff, $G$ is a locally compact topological group, and $X$ is minimal under $G$. Then $X$ is a compact point-distal minimal set if and only if there is a point $x \in X$ such that for any homomorphism $h: X \rightarrow B C(G)$, $h(x)$ is a right weakly distal function.

The proof of this result is similar to the proof of Theorem 4.1. Use is made of Theorem 3.4 instead of Theorem 3.3. We omit the proof.

\section{REFERENCES}

1. J. Auslander and F. Hahn, Point transitive flows, algebras of functions and the Bebutov system, Fund. Math. 60 (1967), 117-137. MR 36 \#4541.

2. L. Auslander and F. Hahn, Real functions coming from flows on compact spaces and concepts of almost periodicity, Trans. Amer. Math. Soc. 106 (1963), 415-426. MR 26 \#1871.

3. J. D. Baum, An equicontinuity condition for transformation groups, Proc. Amer. Math. Soc. 4 (1953), 656-662. MR 15, 51.

4. N. Bourbaki, General topology. Parts 1, 2, Hermann, Paris; Addison-Wesley, Reading, Mass., 1966. MR 34 \#5044a; MR 34 \#5044b.

5. R. Ellis, Distal transformation groups, Pacific J. Math. 8 (1958), 401-405. MR 21 \#96.

6. - - Equicontinuity and almost periodic functions, Proc. Amer. Math. Soc. 10 (1959), 637-643. MR 21 \#5950.

7. Lectures on topological dynamics, Benjamin, New York, 1969.

8. W. H. Gottschalk and G. A. Hedlund, Topological dynamics, Amer. Math. Soc. Colloq. Publ., vol. 36., Amer. Math. Soc., Providence, R.I., 1955. MR 17, 650.

9. A. Knapp, Distal functions on groups, Trans. Amer. Math. Soc. 128 (1967), 1-40. MR 36 \#5923.

10. W. A. Veech, Point-distal flows, Amer. J. Math. 42 (1970), 205-242.

Department of Mathematics, University of Florida, Gainesville, Florida 32601 